\title{
Spatial Dependence Pattern of Energy-Related Carbon Emissions and Spatial Heterogeneity of Influencing Factors in China: Based on ESDA-GTWR Model
}

\author{
Kai Su*(**) and Chienming Lee*† \\ *Natural Resource Management Institute, National Taipei University, Taipei 23741, China \\ **Anxi College of Tea Science, Fujian Agriculture and Forestry University, Fuzhou 350002, China \\ †Corresponding author: Chienming Lee; 2150525002@fafu.edu.cn
}

Nat. Env. \& Poll. Tech.

Website: www.neptjournal.com

Received: 05-02-2020

Revised: $21-02-2020$

Accepted: 02-05-2020

Key Words:

Carbon emissions

Spatial heterogeneity

GTWR

ESDA

Spatial differentiation

\begin{abstract}
To find out the spatial dependence of carbon emissions and its evolution characteristics is the key to achieving regional differential emission reduction strategy. In this study, 30 provinces with different population sizes and in different stages of development in China, were selected to explore the spatial heterogeneity of carbon emissions by exploratory spatial data analysis (ESDA), combined with geographically and temporally weighted regression (GTWR). The findings revealed that (1) energyrelated carbon emissions at the province-level in China increased from 1997 to 2016, with an increment of 8,893 million tons; (2) there is a significant positive spatial correlation between provincial carbon emissions, which showed the characteristics of rising first and then falling; this indicated that provincial carbon emissions have obvious spatial dependent characteristics; (3) the tertiary industry ratio had a restraining effect on carbon emissions, whereas the other three variables, namely GDP, urbanization rate, and energy intensity had a positive effect on carbon emissions of provinces in China; and (4) province-scale spatial differences in and distribution patterns of carbon emissions within the same countrywide, which will help decision making in terms of carbon trading and ecological compensation mechanisms. Therefore, we suggested that in the formulation of reduction policies for carbon emissions, policymakers need to adapt to local conditions which accord to the characteristics of the province.
\end{abstract}

\section{INTRODUCTION}

Global warming largely caused by carbon emissions is a serious problem threatening ecosystems and human development globally (Villoria-Sáez et al. 2016). In its 'Intended Nationally Determined Contributions', China, the top carbon emitter globally (Guan et al. 2009), has committed to reducing carbon dioxide emissions per gross domestic product (GDP) by 60\%-65\% from the 2005 level by 2030 (Mi et al. 2016). As the world's largest developing country, China is in a period of rapid industrialization and urbanization. However, the continuous improvement in living standards has inevitably led to continuous increasing carbon emissions (Shan et al. 2018, Wang \& Zhao 2018). To accomplish the above goal, the Chinese government will face enormous pressure for the next 10-15 years. Due to China's vast territory, there are significant differences in resource endowments, economic development, industrial structure and energy consumption structure between regions, and there are spatial linkages. Thus, it is necessary to find out spatial dependence of provincial energy-related carbon emissions in China, and spatial heterogeneity of its influencing factors, to formulate emission reduction measures in a targeted manner.
Study on carbon emissions in the existing literature mainly focuses on four aspects. (1) Applying the inverted U-curve to explore the carbon emission pathways, the calculation of inflexion points and the applicability of carbon emissions to EKC. For instance, Chen et al. (2019) found that no matter in the eastern, central and western regions in China, the curve relation between carbon emissions and economic development does not meet to EKC hypothesis of inverted U-curve, but there are different inflexion points. Zaidi et al. (2019) took APEC countries as an example, and it is found that there is an inverted " $U$ " type relationship between carbon emissions and economic globalization, and the EKC curve is valid. (2) Using factor decomposition model to explore the driving factors and mechanism of carbon emissions. For instance, the LMDI method was applied to decompose China's household carbon emissions, and the results show that the household consumption structure has the characteristics of high carbonization (Cao et al. 2019). On the other hand, based on the approaches of LMDI and Tapio, the findings of Liang et al. (2019) indicated that the decoupling of carbon intensity from per capita income in the residential building sector of China's four megalopoleis, namely Beijing, Tianjin, Shanghai and Chongqing have 
been implemented. (3) Taking carbon emissions per capita and carbon emission intensity as indicators, the studies analyze the spatial difference, pattern evolution and emission reduction path of carbon emissions. For example, Dong et al. (2018) analysed the curve relationship between land urbanization (LU) and carbon emission intensity (CEI) by using the methods of Diff-GMM and Sys-GMM. (4) The spatial distribution pattern and difference of carbon emission were studied from the perspective of spatial correlation. Yan et al. (2017) analysed the regional difference and spatial correlation of inter-provincial carbon emission efficiency in China, and there is a significant spatial autocorrelation and obvious cluster trend of inter-provincial carbon emission efficiency. Moreover, by analysing the impact of spatial correlation on the geographical and economic matrix, Wang \& He (2019) verifies that the spatial neighbourhood described in the bilateral economic relationship is much superior in trapping the spatial dependence of carbon emissions. In general, the existing literature has made a lot of discussions on carbon emissions and achieved important results, which can be used as a reference for energy-related carbon emissions research. However, most previous studies had seldom considered the spatial dependence of regional carbon emissions, but regard regions as independent and homogeneous individuals. Energy-related carbon emissions are not only influenced by factors such as the industrial structure, economic development level, urbanization and population size of the region, but also the potential correlation between carbon emissions in the surrounding areas. There may be obvious spatial dependence on carbon emissions between regions, and there is spatial heterogeneity of factors affecting carbon emissions. Due to the vast territory and uneven distribution of resources in China, there are great differences in development among provinces. Thus, it is clear that we cannot adopt the policy of cutting at one stroke in dealing with carbon emissions problems.

Furthermore, in the existing academic literature, most researchers used GWR (Geographically Weighted Regression) method to analyze spatial heterogeneity. Although the GWR model considers spatial effects and spatial heterogeneity, it still has some shortcomings. For instance, the GWR model can only consider regression the cross-section data and does not take into account the influence of time effect. GTWR (Geographically and Temporally Weighted Regression) can overcome the above problems effectively. It introduces the time dimension into the model to make the estimation more effective, which provides a new method for the test of spatial heterogeneity. However, the existing literature rarely studies the spatial heterogeneity of the factors affecting carbon emissions based on this method. To address this gap, taking 30 provinces in China as the research object, this study used exploratory spatial data analysis (ESDA) and GTWR model to analyze the spatial heterogeneity of factors affecting energy-related carbon emissions in China. The results can be used as data support for the formulation and implementation of energy-related carbon emission reduction policies in China and provide a reference for other countries/regions, particularly a developing country, to carry out relevant research at the provincial scale.

\section{MATERIALS AND METHODS}

\section{Calculation of Carbon Emissions}

The baseline method provided in the 2006 IPCC Guidelines National Greenhouse Gas Inventories (Eggleston et al. 2006), was used to calculate energy-related provincial carbon emissions in China:

$$
C=\sum_{i=1}^{n} e_{i} \times f_{i} \times k_{i} \times 44 / 12
$$

Where, $C$ represents carbon emissions generated by the consumption of various types of fossil energy; $n$ is fossil energy types; $e_{i}$ is the consumption of certain fossil fuels; $f_{i}$ indicates the standard coal conversion factor, which is used to convert different types of energy into standard coal equivalent; $k_{i}$ is the carbon emission coefficient for different fossil fuels (taken from IPCC reference values), and 44/12 indicates the molecular weight ratio of carbon dioxide to carbon. The calculated parameters for carbon emissions from different types of fossil fuels are given in Table 1.

\section{ESDA}

ESDA mainly uses the methods of statistics, image and chart to analyze the spatial data and explore the spatial relationship of the object of study. Spatial autocorrelation analysis is the main content of ESDA. It aims to study the spatial distribution and correlation of sample objects and reveal the visual phenomenon of spatial dependence and spatial heterogeneity of sample data. Spatial autocorrelation mainly includes global spatial autocorrelation and local spatial autocorrelation.

Table 1: Calculation parameters of carbon emissions for different types of fossil energy.

\begin{tabular}{|lllllllll|}
\hline Energy types & Raw Coal & Coke & Crude Oil & Gasoline & Kerosene & Diesel & Natural Gas & Fuel Oil \\
\hline Coefficient of standard coal $\left(10^{4} \mathrm{tce} / 10^{4} \mathrm{t}\right)$ & 0.7143 & 0.9714 & 1.4286 & 1.4714 & 1.4714 & 1.4571 & 1.3300 & 1.4286 \\
Carbon emission coefficient $\left(10^{4} \mathrm{t} / 10^{4} \mathrm{tce}\right)$ & 0.7559 & 0.8550 & 0.5857 & 0.5538 & 0.5714 & 0.5921 & 0.4483 & 0.6185 \\
\hline
\end{tabular}


1. Global spatial autocorrelation. It mainly reveals the spatial relevance and difference degree of the whole region. Global Moran's $I_{g}$ is an estimation of global spatial autocorrelation statistics, which is expressed as follows:

$$
I_{g}=\frac{\sum_{i=1}^{N} \sum_{j=1}^{N} w_{i j}\left(x_{i}-\bar{x}\right)\left(x_{j}-\bar{x}\right)}{\sum_{i=1}^{N} \sum_{j=1}^{N} w_{i j} \sum_{i=1}^{N}\left(x_{i}-\bar{x}\right)^{2}}
$$

Where, $I_{g}$ is the coefficient of global spatial autocorrelation, its value range is $[-1,1]$; the positive value indicates that the areas with higher (or lower) carbon emissions are spatially significantly agglomerated, and negative values indicate significant spatial differences in carbon emissions levels between the region and its surrounding areas. $n$ is the number of samples, that is, the total evaluation area. $x_{i}$ and $x_{j}$ are observations of spatial positions $i$ and $j$, respectively; $\bar{x}$ is the average value of $x . w_{i j}$ stands for weight, reflecting the degree of influence between the spatial unit of region $i$ and $j$.

2. Local spatial autocorrelation. Global spatial autocorrelation can reveal the spatial dependence of provincial carbon emissions, but it cannot measure the local spatial differences. Thus, the local spatial autocorrelation to measure the degree and significance of the spatial difference between a certain area and its surroundings, and combined with LISA (Local Indicators of Spatial Association) clustering map to analyze the local spatial distribution law. It is expressed as follows:

$$
I_{l}=\frac{\left(x_{i}-\bar{x}\right) \sum_{j=1}^{N} w_{i j}\left(x_{j}-\bar{x}\right)}{\sum_{i=1}^{N}\left(x_{i}-\bar{x}\right)^{2}}
$$

At a given significant level, $I_{l}>0$ indicates that carbon emissions of the observed provinces are similar to those of neighbouring provinces, that is, high-high or low-low; and $I_{l}<0$ indicates that carbon emissions of the observed provinces are different from those of neighbouring provinces (low-high or high-low).

\section{GTWR}

Factors affecting carbon emissions (such as population size, urbanization rate, GDP, energy structure, energy intensity, and industrial structure, etc.) are both unstable and heterogeneous, as well as spatially related. If the traditional regression model is still used to estimate its parameters, it is difficult to reflect the spatial heterogeneity between regions. The GWR (geographically weighted regression) model, initially developed by Brunsdon et al. (1996), which considers the spatial characteristics of the observed variables into the model, revealing the spatial nonstationarity and spatial dependence of the studied variables from a local perspective. Consequently, it is widely used to solve the spatial heterogeneity between units. However, the GWR model can only regress the cross-section data without considering the influence of time factors ( $\mathrm{Li}$ et al. 2019). Thus, it is necessary to embed the time effect into the regression model and construct the geographical and temporally weighted regression (GTWR), which can capture the parameter variation of different spatial units in two dimensions of time and space, so as to make up for the deficiency of GWR model effectively (Huang et al. 2010, Fotheringham et al. 2015). The general formula of GTWR model is as follows:

$$
y_{i}=\beta_{0}\left(u_{i}, v_{i}, t_{i}\right)+\sum_{k=1}^{d} \beta_{k}\left(u_{i}, v_{i}, t_{i}\right) x_{i k}+\varepsilon_{i}, i=1,2, \ldots, n ; k=1,2, \ldots, d \ldots
$$

Where, $y_{i}$ is the interpreted variable of the $i$-th sample point; $u_{i}, v_{i}$, and $t_{i}$ represent the longitude coordinates, latitude coordinates, and time coordinates of the $i$-th sample point, respectively. $\left(u_{i}, v_{i}, t_{i}\right)$ represents the space-time dimension coordinates of the $i$-th sample point; $B_{0}\left(u_{i}, v_{i}, t_{i}\right)$ is the constant term of the $i$-th sample point; $\beta_{k}\left(u_{i}, v_{i}, t_{i}\right)$ denotes the regression coefficient of the $k$-th explanatory variable at the $i$-th sample point. $x_{i k}$ is the $k$-th explanatory variable of the $i$-th sample point; $\varepsilon_{i}$ indicates a random error.

\section{Data Source and Variables Selection}

Based on referring to the above-related research results, this study selects four indicators GDP(G), Urbanization rate (U), Tertiary industry ratio (S) and Energy consumption per unit of GDP(E) to measure the provincial energy-related carbon emissions from the four aspects of economic development level, population composition, industrial structure and energy intensity, respectively. To reduce the heteroscedasticity of spatial data in the model, the data of each variable were processed by natural logarithm at first, which were identified as $\ln G, \ln U, \ln S$ and $\ln E$, respectively.

Energy-related carbon emissions of each province in 1997-2016 were calculated by equation (1). The data of socio-economic and energy consumption in 30 provinces (Tibet, Hong Kong, Taiwan and Macao are not included due to the lack of data) were all from National Bureau of Statistics of China (http://data.stats.gov.cn/english/). Besides, this paper set 1997 as the benchmark year, so GDP and energy intensity were converted to constant prices to avoid the impact of inflation. Additionally, the base map comes from Data Centre for Resources and Environmental Sciences of the Chinese Academy of Sciences (www.resdc.cn). 


\section{RESULTS AND ANALYSIS}

\section{Energy-Related Carbon Emissions of Provinces}

According to equation (1), cumulative carbon emissions of each province in China from 1997 to 2016 were listed in Table 2.

From 1997 to 2016, the three provinces in China with the highest cumulative carbon emissions (Table 2) were Shandong $\left(16061 \mathrm{M}_{\mathrm{t}}\right)$, Hebei $\left(12730 \mathrm{M}_{\mathrm{t}}\right)$ and Shanxi $\left(11972 \mathrm{M}_{\mathrm{t}}\right)$, accounting for $23.80 \%$ of the cumulative carbon emissions in China, which has significantly contributed to national carbon emissions. The three cities with the lowest cumulative carbon emissions were Ningxia $\left(2115 \mathrm{M}_{\mathrm{t}}\right)$, Hainan (716 $\mathrm{M}_{\mathrm{t}}$ ) and Qinghai $\left(696 \mathrm{M}_{\mathrm{t}}\right)$. Their total accumulated carbon emissions were $3527 \mathrm{M}_{\mathrm{t}}$, accounting for only $2.06 \%$ of the nation's cumulative carbon emissions over the study period. Each province's carbon emissions tend to be closely related to the region's economy and industrial structure. For example, by the end of 2016, the comprehensive energy consumption of Shandong, Hebei and Shanxi reached $62356.44 \times 10^{4}$, $39912.13 \times 10^{4}$ and $39146.06 \times 10^{4} \mathrm{t}$, respectively, accounting for $10.76,6.89$, and $6.75 \%$ of the total energy consumption above the designated size in China.

To reveal the spatial characteristics of energy-related carbon emissions at the province scale in China, the calculated data of carbon emissions were classified and archived. The Natural Breaks Method was used to cluster the carbon emissions of each province by ArcGIS10.7, and the corresponding spatial distribution is shown in Fig.1.

From 1997 to 2016, the medium-high and high emission areas moved to coastal provinces from inland areas, which were related to the urbanization and industrialization of coastal provinces. For instance, due to urban infrastructure construction, coupled with urbanization and industrialization, the socio-economic development in Jiangsu province has further accelerated in recently; this has led to an increase in fossil energy consumption, which in turn has boosted its carbon emissions. In contrast, Liaoning, Jilin and Heilongjiang, which are located in northeast China, have a lower level with respect to economic development in recent years; therefore, there was a downward trend of its carbon emissions. Additionally, it is worth pointing out that Beijing, as the capital of China, its carbon emissions peaked in 2010 , reaching $138.61 \mathrm{M}_{\mathrm{t}}$; then declined by $29.2 \%$ in 2016 , at $107.34 \mathrm{M}_{\mathrm{t}}$. The primary cause for the reductions is due to its technological progress, developed tertiary industry (e.g. modern service industry and financial industry) and emissions reduction policies. Data indicated that in 2018, Beijing's primary industry, secondary industry and tertiary industry accounted for $0.4 \%, 18.6 \%$ and $81.0 \%$, respectively; among them, the financial industry has become the first pillar industry, effectively reducing its consumption of fossil energy.

Table 2: Cumulative carbon emissions at the province scale in China during 1997-2016 (Million tons, $\mathrm{M}_{\mathrm{t}}$ ).

\begin{tabular}{|llllllllll|}
\hline Beijing & Tianjin & Heibei & Shanxi & Inner Mongolia & Liaoning & Jilin & Heilongjiang & Shanghai & Jiangsu \\
\hline 2349 & 2872 & 12730 & 11972 & 8992 & 10925 & 4042 & 5685 & 4627 & 10722 \\
Zhejiang & Anhui & Fujian & Jiangxi & Shandong & Henan & Hubei & Hunan & Guangdong & Guangxi \\
6481 & 5188 & 3316 & 2790 & 16061 & 9114 & 5613 & 4587 & 8816 & 2714 \\
Hainan & Chongqing & Sichuan & Guizhou & Yunan & Shaanxi & Gansu & Qinghai & Ningxia & Xijiang \\
716 & 2329 & 5213 & 4104 & 3529 & 5239 & 2962 & 696 & 2115 & 4811 \\
\hline
\end{tabular}
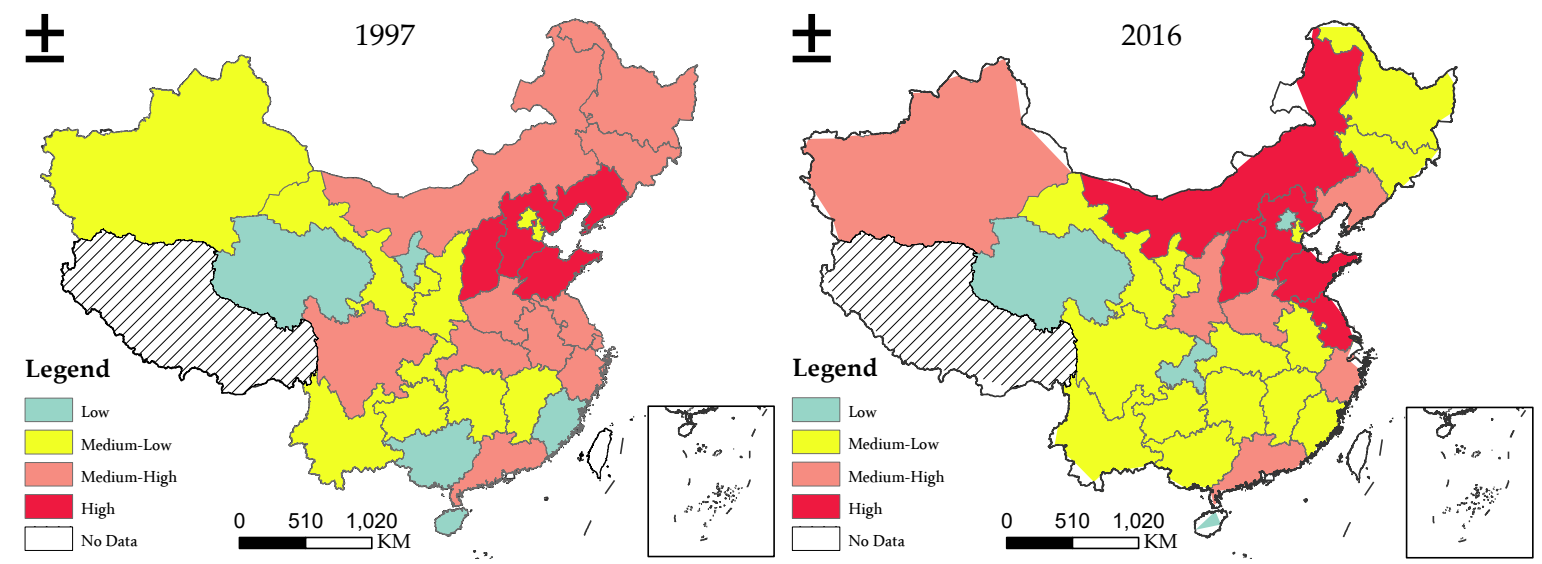

Fig.1: The spatial layout of carbon emissions at the province scale in China. 


\section{Global Spatial Dependence Pattern of Provincial Energy-Related Carbon Emissions}

This study used ArcGIS10.7 to conduct spatial autocorrelation analysis on carbon emissions data of 30 provinces in China from 1997 to 2016. The variation curves of Moran's $I$ index and P-value for each year were shown in Fig. 2.

As can be seen from Fig. 2, the Moran's $I_{g}$ index from 1997 to 2016 is positive and all passed the significance test at the level of 5\%, indicating that the spatial distribution of provincial carbon emissions in China is not completely random, but has significant spatial dependence characteristics. In other words, provinces with relatively high carbon emissions tend to be adjacent to other provinces with high carbon emissions, while provinces with relatively low carbon emissions tend to be adjacent to other provinces with low carbon emissions. From the perspective of the change process, the spatial correlation trend of provincial carbon emissions showed a pattern of rising and then decreasing. From 1997 to 2008, the Moran's $I_{g}$ index showed an upward trend, indicating that the spatial dependence of provincial-scale carbon emissions was increasing, reaching the highest value of 0.27 in 2008 . Since 2008, Moran's $I_{g}$ index has shown a fluctuating downward trend, indicating that the spatial concentration effect of provincial carbon emissions in China is weakening as a whole.

The above changes in the Moran's $I_{g}$ index showed that the spatial dependence of provincial-scale carbon emissions in China mainly occurs at four-time nodes, namely 1997 , 2008, 2009 and 2016. Therefore, the following contents mainly focus on these four-time nodes for further analysis.

\section{Local Spatial Dependence Pattern of Provincial Energy-Related Carbon Emissions}

The global Moran's $I_{g}$ index can only explain the overall spatial dependence of carbon emissions at the provincial scale in China, however, it cannot represent the specific structure and spatial correlation of spatial dependence of provincial carbon emission. Hence, according to the spatial and temporal distribution characteristics of different provinces, representative years (1997, 2008, 2009, 2016) were selected for comparative study, and LISA clustering map of four years were drawn by using ArcGIS 10.7 (Fig. 3).

As shown in Fig. 3, at a significant level of 5\%, the local spatial dependence of carbon emissions at the provincial scale in China is relatively obvious. There are two significant regions in the northwest and central China, suggesting that there is a positive spatial effect between provinces, which makes the provinces and the surrounding areas show the development trend of mutual connection and interaction. It is mainly reflected in the small spatial difference, the region with high carbon emissions in the region itself and surrounding provinces (High-High), mainly concentrated in Hebei, Shandong, Henan and Anhui. The reason is that these provinces have a good industrial foundation, a developed heavy chemical industry and abundant energy resources. The rapid progress of industrialization consumes a large amount of fossil energy, resulting in strong regional spatial correlation and interactive spillover. Moreover, Hebei, Shandong, Henan and Anhui have reached significant levels in four periods. The spatial difference is large, and the regional carbon emissions are higher, but the lower average areas (High-Low) are mainly distributed in Xinjiang. Although the spatial difference is small, the region itself and the surrounding average lower region (Low-Low), all provinces have not passed significant tests. In terms of the overall number, the number of High-High types of provinces has been increasing since 1997, which is consistent with the upward trend of global Moran's $I_{g}$ values

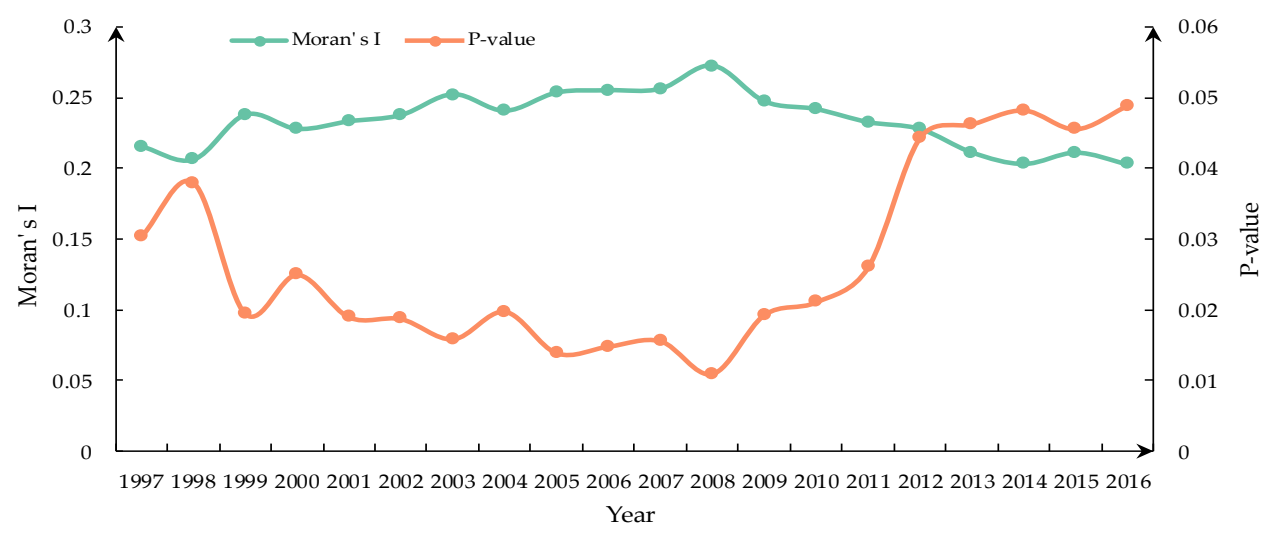

Fig.2: Global Moran's I index of provincial carbon emissions in China from 1997 to 2016. 


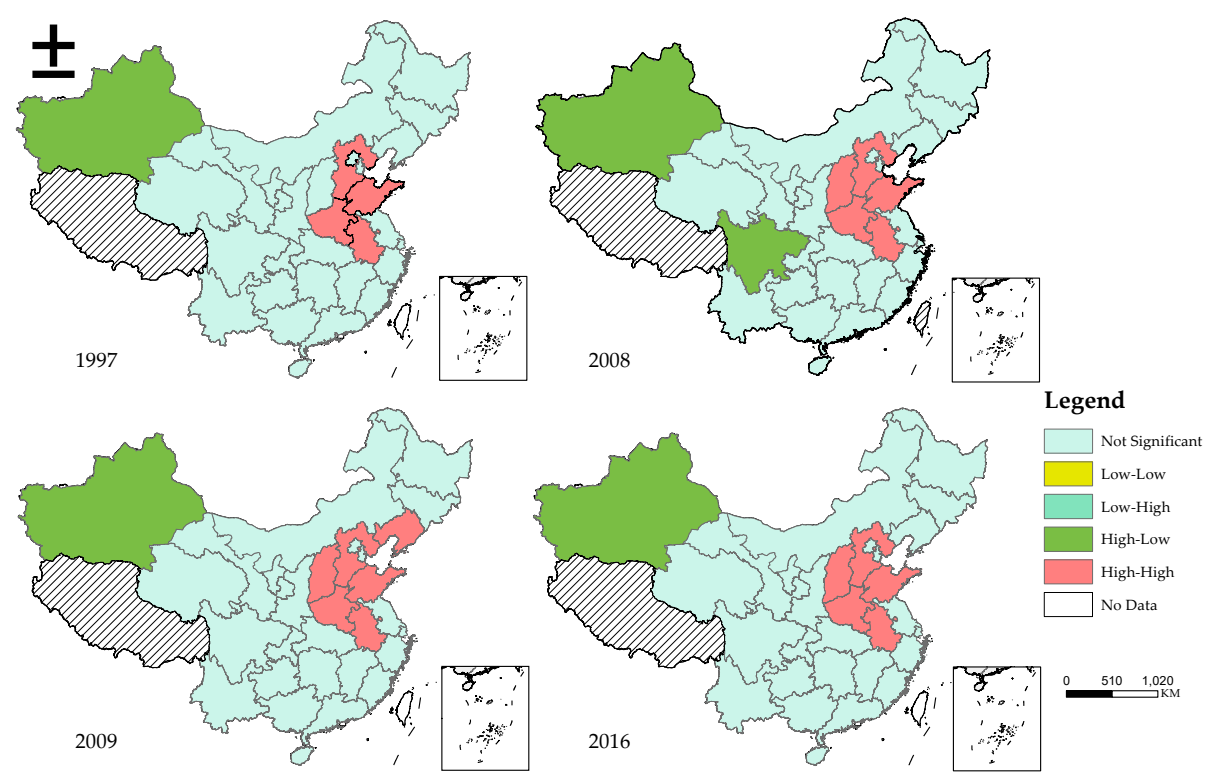

Fig. 3: LISA clustering map of provincial carbon emission in China.

from 1997 to 2008. Thus, the diffusion effect of carbon emissions reduction is gradually increasing.

\section{Spatial Heterogeneity of Influencing Factors of Provincial Carbon Emissions}

The above ESDA analysis showed that the spatial distribution of carbon emissions in China is significantly spatially dependent. To analyze the distribution of each influencing factor more clearly, it is necessary to apply the GTWR model for measurement. This study mainly used ArcGIS 10.7 to realize the coefficient estimation of GTWR model based on the attributes of time and space. The estimation results of the regression coefficient calculated by GTWR were listed in Table 3, while the coefficient distribution of each influencing factor in 1997 and 2016 are shown in Fig. 4.

As can be seen from Table 3, the value of $\mathrm{R}^{2}$ and adjusted $\mathrm{R}^{2}$ of GTWR were 0.995981 and 995956 , respectively, indicating that the GTWR model can better explain the influence of independent variables (influencing factors) on dependent variables (carbon emissions), and better explain the data with spatio-temporal characteristics. This means the GTWR's coefficients are more in line with real-world interpretations. Accordingly, the distribution characteristics of each influencing factor in different temporal and spatial dimensions are shown in Fig. 4.

\section{Spatio-Temporal Variation of Economic Development (Lng) on Carbon Emissions}

It can be seen from Fig. 4 that GDP has an obvious influence on carbon emissions and is positively correlated with carbon emissions, indicating that GDP growth is a key factor in increasing carbon emissions. The regression coefficients of Xinjiang, Qinghai and Liaoning are larger, while the provinces with smaller coefficients are Sichuan, Fujian and Zhejiang. This showed that the differences in economic development between different provinces in China are quite prominent. Therefore, when determining the countermeasures for carbon emissions reduction in each province, the implementation of policies should be determined according to the specific economic development stage of each province. Moreover, carbon emissions have increased rapidly in the process of economic growth in areas with low economic development level in the western areas of China, such as provinces of Qinghai and Xinjiang. Therefore, at this stage, the government should focus on transforming the economic structure and introducing low-energy industries from the developed eastern regions to reduce the growth of carbon emissions.

Table 3: Estimation results of regression coefficient calculated by GTWR.

\begin{tabular}{|llllllll|}
\hline Bandwidth & Residual Squares & Sigma & AICc & $\mathrm{R}^{2}$ & adjusted $\mathrm{R}^{2}$ & Spatio-temporal Distance Ratio & Trace of SMatrix \\
\hline 0.114996 & 6.25916 & 0.098894 & -991.525 & 0.995981 & 0.995956 & 0.273068 & 67.1389 \\
\hline
\end{tabular}


Additionally, carbon emissions growth rate in Sichuan, Hainan and Guangdong has slowed down in the process of economic growth, which requires the government to take policy measures to bring it to an inflexion point in terms of technological upgrading of energy efficiency and low carbonation of the industrial structure. Overall, China's provincial GDP growth will exacerbate carbon emissions during the study period, while other conditions remain unchanged.

\section{Spatio-Temporal Variation of Population Composition (Lnu) on Carbon Emissions}

The $\ln U$ estimation coefficient reflects the contribution of different regional urbanization rates to the carbon emissions growth, and the spatial spillover effect of urbanization rates in neighbouring provinces.

As can be seen from Fig. 4, the high-value areas of regression coefficient of urbanization rate are concentrated in Xinjiang, Qinghai, Gansu, showing a certain gradient distribution. This indicated that in these western regions where industrial-oriented industries are being promoted, most of the employed labour force is transferred to the industrial industry in the context of increasing urbanization rate, causing an increase in the scale of carbon emissions. The low values of the regression coefficient of the urbanization rate are concentrated in the central region, which has a relatively high degree of urbanization. However, due to its advanced economy and technology, the improvement of the urbanization rate is not the most important reason affecting the carbon emission of these regions. However, it should be noted that the increase in urbanization rate does not mean that it always promotes carbon emission growth. Some scholars believe that there is an inverted "U -shaped" relationship between urbanization level and carbon emissions (Ehrhardt-Martinez et al. 2002, York et al. 2003). This means that the overall development of urbanization still plays a role in promoting carbon emissions when the inflexion point of urbanization inverted " $U$ " curve is not reached; however, when it reaches the inflexion point, the urbanization level has a slowing effect on carbon emissions.

\section{Spatio-Temporal Variation of Industrial Structure (Lns) on Carbon Emissions}

The industrial structure is an important factor that influencing carbon emissions. The results indicated that the tertiary/ service industry had a reduced effect on carbon emissions in most China's provinces, and there are regional differences in the influence of industrial structure on carbon emissions (Fig. 4). Judging from the regression coefficients, the regression coefficient between the tertiary industry ratio and carbon emissions showed an increasing trend from west to east of China. Among them, the provinces with greater impact on carbon emissions from the industrial structure are Hubei, Henan, Zhejiang and Fujian in central and eastern China, while the provinces with relatively smaller impact are Gansu, Qinghai, Xinjiang and Guizhou in northwest China. This means that carbon emissions from provinces, located in the eastern coastal area, are more vulnerable to the industrial structure than in the inland western regions. The reason may be related to the differences in the original economic structure and the different ideas of industrial development in different provinces, which may lead to the more regional characteristics of the local industrial structure.

In general, except that tertiary industry ratio (S) had an inhibitory effect on carbon emissions in Fujian's cities, the other three variables (GDP, urbanization rate, and energy consumption per unit of GDP) had a promoting effect on carbon emissions. This means that with accelerating urbanization and industrialization, the energy consumption of China's provincial areas is continuously increasing. If the principle of 'business as usual 'is followed, the carbon emissions in China's provincial areas has an increasing trend; in contrast, if the proportion of low-carbon industries, such as the service industry, is further increased, energy efficiency will be further improved through technology, thereby reducing energy intensity. The growth of carbon emissions in various provinces of China therefore had a certain inhibitory effect. In short, the development of the tertiary industry should be encouraged, as it can reduce carbon emissions and environmental pollution and save the land.

\section{Spatial-Temporal Variation of Energy Intensity (Lne) on Carbon Emissions}

Energy intensity reflects the contribution of technological progress in different regions to their own carbon emissions growth. According to the results, the coefficients of energy intensity's impact on provincial carbon emissions in China are positively correlated, and there are significant spatial differences. This suggested that energy intensity can promote carbon emissions, and there is a significantly promote carbon emissions in different provinces. Compared with other factors, energy intensity has the least effect on carbon emissions, which is mainly concentrated in the western regions of China such as Xinjiang, Gansu and Qinghai, and is gradually increasing to the southeast, according to the data. This is mainly because the eastern region of China is rich in resources and in a period of rapid industrialization, which will inevitably increase its energy intensity, thus increasing the pressure on carbon emissions reduction.

In a word, the provinces with higher technical level and better economic conditions in eastern China, energy intensity has a relatively large impact on the distribution 

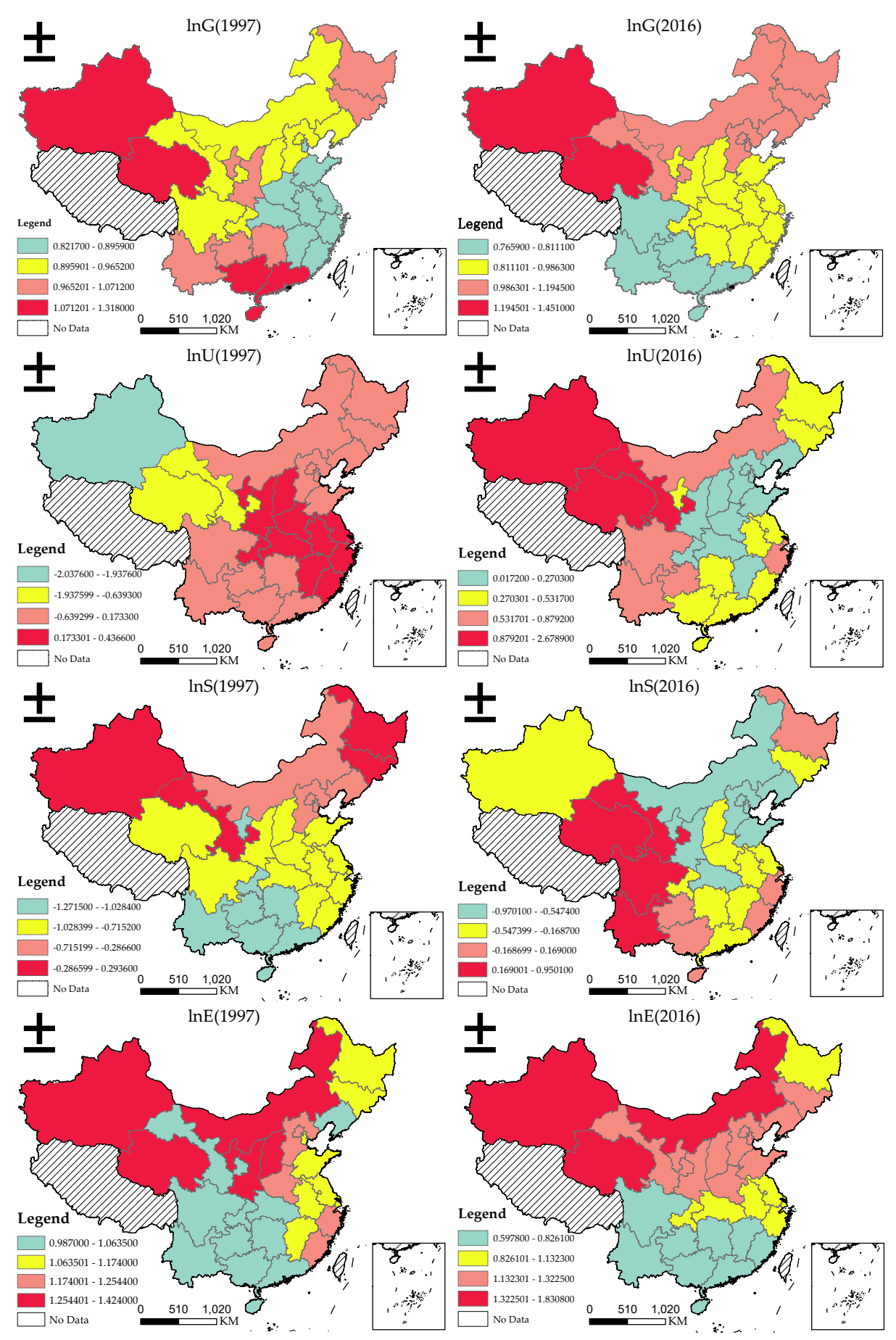

Fig. 4: Spatial distribution of regression coefficients calculated by GTWR model in 1997 and 2016.

of provincial carbon emissions. On the other hand, in the western provinces of China with lower technical level and relatively poor economic conditions, energy intensity has a relatively small impact on carbon emissions. Hence, to achieve carbon emissions reduction targets, the government needs to improve energy efficiency by introducing energy conservation and emission reduction technologies, according to the low carbon development model in different regions. 
These measures can achieve the dual objectives of controlling carbon emissions and reducing carbon intensity.

\section{DISCUSSION AND CONCLUSIONS}

Considering the deficiency of past literature study on spatial heterogeneity of factors affecting carbon emissions, this study introduced geographically and temporally weighted regression (GTWR) into the spatial analysis of carbon emissions, which provides a new approach to spatial heterogeneity testing. Based on the provincial panel data of China from 1997 to 2016, 30 provinces with different population sizes and in different stages of development in China, were selected to analyze the influencing factors of carbon emissions by exploratory spatial data analysis (ESDA) combined with GTWR method. Thus, the major conclusions are summarized herein and put forward targeted policy recommendations accordingly.

Energy-related carbon emissions at the province level in China had increased by $215.08 \%$ from 1997 to 2016, with an increment of $8893 \mathrm{M}_{\mathrm{t}}$. Provinces such as Anhui, Jiangsu, and Hebei have high-level carbon emissions, while Guizhou, Qinghai and Hainan on the contrary. These results suggest that the government formulate corresponding incentive measures or economic incentives to encourage enterprises to accelerate the energy-saving technological transformation and improve energy efficiency. Therefore, it is necessary to unswervingly promote the existing energy conservation and emission reduction policies and measures to achieve China's further emission reduction targets, including financial incentives for energy conservation technology transformation, as well as the mandatory target of provincial energy intensity (Du et al. 2018).

From 1997 to 2016, the global Moran's I index has a positive value and showed a trend of rising first and then falling, and all passed the 5\% significance test. This indicated that provincial carbon emissions in China have a significant positive spatial correlation, that is, provinces with higher or lower carbon emissions tend to be spatially adjacent. Moreover, there is a positive spatial effect between provinces, which makes the development trend of mutual relationship and interaction between provinces and surrounding areas; therefore, carbon emissions are affected by different geographical locations, according to LISA clustering map of local autocorrelation. Also, the regression results of the GTWR model indicated that the influencing factors of carbon emissions have obvious spatial heterogeneity. The findings revealed that tertiary industry ratio had a restraining effect on the carbon emissions, whereas the other three variables, namely, GDP, urbanization rate and energy intensity had a positive effect on carbon emissions of provinces in China.
These results suggest that in the formulation of reduction policies for energy-related carbon emissions, policymakers need to adapt to local condition according to the characteristics of the province. Specifically, for the north-western provinces of China with low economic development (e.g. Guizhou, Gansu, Qinghai, etc.), the government should focus on improving energy efficiency and improving energy consumption structure; meanwhile, the government should further readjust and optimize industrial structures and develop low-carbon industries such as high-tech and service industries. On the other hand, for the economically developed eastern and southern coastal provinces (e.g. Shanghai, Zhejiang, Guangdong, and Jiangsu, etc.), the government should focus on new technologies, green energy, and modern services; meanwhile, it will build an economic structure transformation and upgrading that promotes low-carbon development across provinces, thereby forming a reduction path for coordinated economic and environmental development. Furthermore, the government should strengthen the guidance of low-carbon policy in energy-rich areas such as Hebei, Shanxi, etc. The dual reduction targets of carbon emissions and energy intensity will be incorporated into the local government assessment system, and policymakers need to implement the accountability system for carbon emissions reduction.

Additionally, this study revealed a province-scale spatial difference in and distribution pattern of carbon emissions within the same countrywide, which will aid decision making in terms of carbon trading and ecological compensation mechanisms. The results may provide policymakers with insight into spatial patterns of carbon emissions among provinces when macro-control policies are formulated. Our study suggested that inland provinces' overall carbon emissions levels were lower than those of coastal provinces in China. Apart from different provinces being stages of economic development, this is likely mainly due to their various natural environments and resource. The coastal areas of China are economically developed and highly dependent on resources from inland areas. Further development in such areas is often constrained by carbon emission quotas. Therefore, they have the option to buy additional carbon emission credits from other regions, since it is more expensive for them to cut emissions than it is for inland regions. Some inland areas of China, such as Yunnan, Guangxi and Sichuan, etc., are mostly located in mountainous areas with rich ecological resources, especially in terms of the forest. Forestry ecosystem has carbon storage and sequestration functions making these regions large carbon sinks and provide surplus carbon emissions quotas for carbon trading if needed. Our results can thus provide helpful information and accurate data for formulating policies to promote the formation of a beneficial interactive spatial pattern of countrywide carbon emissions. 
Targets can be realized by establishing carbon emission trading system as ecological compensation for implementing green development strategies to increase ecosystem service in inland areas, from which the coastal areas in China can purchase carbon emission quotas.

It is worth mentioning here that our studies aimed to address a gap in relevant research. To a certain extent, this study revealed the spatial heterogeneity of driving factors affecting energy-related carbon emissions, which can provide data support for the formulation of carbon emission reduction strategies. However, the spatial interaction effect between driving factors can also affect the spatial differentiation pattern of carbon emissions. Hence, efforts to explore the correlation between factors by using Geodetector method should be seriously considered in future research.

\section{ACKNOWLEDGMENTS}

The authors gratefully acknowledge the financial support from the Natural Science Foundation of Fujian Province, China (Grant No. 2018J01652).

\section{REFERENCES}

Brunsdon, C., Fotheringham, A.S. and Charlton, M.E. 1996. Geographically weighted regression: a method for exploring spatial nonstationarity. Geogr. Anal., 28: 281-298.

Cao, Q., Kang, W., Xu, S., Sajid, M. J. and Cao, M. 2019. Estimation and decomposition analysis of carbon emissions from the entire production cycle for Chinese household consumption. Journal of Environmental Management, 247: 525-537.

Chen, Yu., Zhao, J.C., Lai, Z.Z., Wang, Z. and Xia, H.B. 2019. Exploring the effects of economic growth, and renewable and non-renewable energy consumption on China's $\mathrm{CO}_{2}$ emissions: Evidence from a regional panel analysis. Renew. Energ., 140(2): 341-353.

Du, Q., Lu, X., Li, Y., Wu, M., Bai, L. and Yu, M. 2018. Carbon emissions in China's construction industry: calculations, factors and regions. Int. J. Environ. Res. Public Health., 15(6): 1220.

Dong, F., Bian, Z., Yu, B., Wang, Y., Zhang, S., Li, J., Su, B. and Long, R. 2018. Can land urbanization help to achieve $\mathrm{CO}_{2}$ intensity reduction target or hinder it? Evidence from China. Resour. Conserv. Recy., 134: 206-215.
Eggleston, H.S., Buendia, L., Miwa, K., Ngara, T. and Tanabe, K. 2006. 2006 IPCC Guidelines for National Greenhouse Gas Inventories. Institute for Global Environmental Strategies, Kanagawa, Japan, Intergovernmental Panel on Climate Change.

Ehrhardt-Martinez, K., Crenshaw, EM. and Jenkins, J.C. 2002. Deforestation and the environmental Kuznets curve: A cross-national investigation of intervening mechanisms. Soc. Sci. Q., 83(1): 226-243.

Fotheringham, A.S., Crespo, R. and Yao, J. 2015. Geographical and temporal weighted regression (GTWR). Geogr. Anal., 47(4): 431-452.

Guan, D., Peters, G.P., Weber, C.L. and Hubacek, K. 2009. Journey to world top emitter: An of the driving forces of China's recent $\mathrm{CO}_{2}$ emissions surge. Geophys. Res. Lett., 36 (4): 1-5.

Huang, B. Wu, B. and Barry, M. 2010. Geographically and temporally weighted regression for modeling spatio-temporal variation in house prices. Int. J. Geogr. Inf. Sci., 24: 383D401.

Li, M.J., Wang, J. and Chen. Y.H. 2019. Evaluation and influencing factors of sustainable development capability of agriculture in countries along the Belt and Road Route. Sustainability, 11: 2004.

Liang, Y., Cai, W. and Ma, M. 2019. Carbon dioxide intensity and income level in the Chinese megacities' residential building sector: Decomposition and decoupling analyses. Sci. Total. Environ., 677: 315-327.

Mi, Z., Zhang, Y., Guan, D., Shan, Y., Liu, Z., Cong, R., Yuan, X.C. and Wei, Y.M. 2016. Consumption-based emission accounting for Chinese cities. Appl. Energy., 184: 1073-1081.

Shan, Y., Guan, D., Zheng, H., Ou, J., Li, Y., Meng, J., Mi, Z., Liu, Z. and Zhang, Q. 2018. China $\mathrm{CO}_{2}$ emission accounts 1997-2015. Scientific Data, 5(1): 1-14.

Villoria-Sáez, P., Tam, V.W.Y., Merino, M.D.R., Arrebola, C.V. and Wang, X. 2016. Effectiveness of greenhouse-gas Emission Trading Schemes implementation: A review on legislations. J. Clean. Prod., 127: 49-58.

Wang, Y. and Zhao, T. 2018. Impacts of urbanization-related factors on $\mathrm{CO}_{2}$ emissions: evidence from China's three regions with varied urbanization levels. Atmos. Pollut. Res., 9(1): 15-26.

Wang, Y.Y. and He, X.B. 2019. Spatial economic dependency in the environmental Kuznets curve of carbon dioxide: The case of China. J. Clean. Prod., 218: 498-510.

Zaidi, S.A.H., Zafar, M.W., Shahbaz, M. and Hou, F. 2019. Dynamic linkages between globalization, financial development and carbon emissions: Evidence from Asia Pacific economic cooperation countries. J. Clean. Prod., 228: 533-543.

York, R., Rosa, E.A. and Dietz, T. 2003. STIRPAT, IPAT and ImPACT: Analytic tools for unpacking the driving forces of environmental impacts. Ecol. Econ., 46: 351-365.

Yan, D., Lei, Y., Li, L. and Song, W. 2017. Carbon emission efficiency and spatial clustering analyses in China's thermal power industry: Evidence from the provincial level. J. Clean. Prod., 156: 518-527. 